\title{
Why energy lossless ferromagnetic materials can add energy losses onto type-ll superconductors?
}

Harold Ruiz ( $\nabla$ dr.harold.ruiz@leicester.ac.uk)

University of Leicester

Muhammad Fareed

University of Leicester

\section{Research Article}

Keywords: SFM, NiZn ferrites, hysteretic, SC-SFM, SC

Posted Date: January 12th, 2021

DOI: https://doi.org/10.21203/rs.3.rs-141275/v1

License: (c) (i) This work is licensed under a Creative Commons Attribution 4.0 International License. Read Full License 


\title{
Why energy lossless ferromagnetic materials can add energy losses onto type-II superconductors?
}

\author{
M. U. Fareed ${ }^{1}$ and H. S. Ruiz ${ }^{1, \dagger}$ \\ ${ }^{1}$ School of Engineering, University of Leicester, University Rd, Leicester LE1 7RH, United Kingdom \\ †dr.harold.ruiz@leicester.ac.uk
}

\begin{abstract}
Understanding the physical coupling between the macroscopic electromagnetic properties of type II superconductors (SC) and soft ferromagnetic materials (SFM), is root for progressing onto the application of SC-SFM metastructures in scenarios such as magnetic cloaking, magnetic shielding, and loss free current transmission systems. However, in the latter case understanding the origin of the rise in the hysteresis losses of the superconductor by effect of the coupling with the SFM has historically resulted in a notable challenge, it because this rise in the AC losses is simply counterintuitive due to the fact that the SFM itself does not add magnetization losses to the system and furthermore, there is no evidence of electrical current sharing between these two materials. Thus, aimed to resolve this long-standing problem, in this paper, we present a semi-analytical model for monocore SC-SFM heterostructures of cylindrical cross-section and self-field conditions, showing the first known map of AC-losses for SC-SFM magnetically shielded wires, with magnetic relative permeabilities for the SFM ranging from $m u_{r}=5$ (NiZn ferrites) to $m u_{r}=350000$ (pure Iron). The distribution of current density and magnetic field inside the SC-SFM metastructure is shown in great detail, revealing a remarkable agreement with the intriguing magneto optical imaging observations that were originally questioning the validness of the critical state theory. In this sense, we have extended the critical state theory within its variational formalism, incorporating a multipole functional approach which allows the direct finding of the coupling terms between a SC current and a SFM sheath, proving that all reported phenomena for the self-filed hysteretic behavior of SC-SFM heterostructures can be understood within the classical critical state model without the need to recur to the ansatz of overcritical currents.
\end{abstract}

\section{Introduction}

Due to the novel phenomena and applications that can be envisaged by the use or devising of metamaterials, the developing of Superconducting-Ferromagnetic heterostructures have been the focus of considerable attention in recent years. ${ }^{1-11}$ Particular focus has been played to the role of magnetization and demagnetization properties of these kind of systems ${ }^{11,12}$, as well to the study of their magnetic cloaking features ${ }^{4-8}$, and the shielding properties of type II superconductors (SC) surrounded or in the near proximity of a soft ferromagnetic material (SFM) ${ }^{13-22}$. However, the influence of the physical coupling between the macroscopic electromagnetic properties of these materials on the overall hysteresis losses of SC/SFM heterostructures for AC applications is yet to be understood.

Several semi-analytic approaches for the understanding of magnetic shielding properties of type-II superconducting materials surrounded by soft high permeability magnets of various configurations have been already proposed by Genenko et. al., including infinitely thin superconducting strips ${ }^{23-31}$, cylindrical tubes ${ }^{5,32,33}$, and finite type-II HTS cylindrical filaments coated by a SFM sheath ${ }^{12,34,35}$. However, a direct inclusion of the magnetoestaic coupling between the profiles of current inside a type II superconductor of finite size and the SFM sheath is not directly included, which hinders the actual mechanisms that give rise to an increase of the AC losses inside the SC material by the action of the SFM. In fact, a theoretical explanation for the increment of the AC losses in monocore SC-SFM heterostructures at self-field conditions, i.e., under applied transport current but no external magnetic field, has not been reached for even the simplest configuration of a SC-SFM wire of cylindrical cross section, a problem that has remained open for about two decades ${ }^{36-38}$.

Moreover, an intriguing and yet unexplained modification of the magnetic flux distribution within the SC core of Iron sheathed $\mathrm{MgB}_{2}$ monocore wires has been experimentally observed by Magneto Optical Imaging techniques, which from the indirect calculation of the critical current density by magnetization measurements ${ }^{39-41}$, it was initially thought that the local deformation in the magnetic flux was caused by the occurrence of the somehow exotic overcritical current densities at the flux-free regions, similar to the concept of overcritical currents originally introduced for infinitely thin strips in the proximity of a $\mathrm{SFM}^{24}$, i.e, where apparently the SC can develop regions where without introducing additional pinning centres, the critical state Bean's law, $J \leq J_{c 0}$, with $J_{c 0}$ the critical current density at self-field conditions, is violated without destroying the SC state. Nevertheless, although it is true that the shielding properties of the $\mathrm{SFM}$ can enhance the critical current density of $\mathrm{MgB}_{2}-\mathrm{Fe}$ 
wires ${ }^{42}$, as $\mathrm{MgB}_{2}$ is known to show a magnetic field dependence of the critical current density $J_{c}(H)$ that for perpendicular magnetic fields decreases as $H$ increases ${ }^{19}$, these overcritical current densities have not been directly observed by magneto optical imaging methods ${ }^{41,43,44}$, neither by direct transport critical current density measurements ${ }^{18}$, precluding therefore their existence (at least) in this geometry. However, certain amount of magnetic field is observed in regions where no transport current is expected to flow under the critical state regime for bare SC at self-field conditions and also, a significant rise and drop of the local magnetic field within the SC core near the surface of the SFM sheath has been observed ${ }^{41}$, both being aspects that are intuitively in disagreement with the critical state theory and which have been remarkably challenging to convey within this qualitative framework, this despite the largely recognized success that from fundamental physical principles the critical state theory has reached for all known type-II superconductors ${ }^{45,46}$.

These problems have been somehow ignored not only due to the engineering prospects of reducing the AC losses in multifilamentary superconductors by the magnetic screening effect of the SFM coatings, but more importantly due to the intrinsic difficulties to determine the physical mechanism that from the macroscopical point of view couples the electromagnetic properties of SCs and SFMs at a local level, i.e., inside both materials. Even in the most ideal of the cases, a perfectly cylindrical type II SC wire of infinite length obeying the general critical state model ${ }^{45}$, where a fully analytic solution for the time dynamics of the flux-front profiles exists, i.e, circular patterns of current density under self-field conditions (See Fig. 1), and where an analytic equation for the calculation of the AC-losses can be consequently derived ${ }^{1,47}$, it results apparently impossible to determine the cause of the intriguing increment of the AC-losses when the SC is embedded within a closed SFM sheath if the fundamental critical state model is adopted. The cause of this is threefold: Firstly, the calculation of the AC losses inside a SC is mainly determined by the distribution of profiles of current density, which results the same regardless if the SC is embedded within a fully closed SFM sheath or not; Secondly, the fact that the distribution of magnetic field outside the cylindrical SC-SFM heterostructure under self-field conditions is basically the same than the one of just the SC cylindrical wire, with no apparent change in the critical current density of the SC by the influence of the SFM, regardless of its magnetic permeability ${ }^{23}$, makes no reason to think that under this geometry the electric or magnetic field patterns inside the SC material changes, therefore making really difficult to understand what could be the possible cause for an increment of the AC-losses, when the SFM is not the source of these hysteresis losses, and; Thirdly, finite element methods based on the solution of the partial differential equations systems defined by Maxwell Equations, which commonly solve a global system including the surroundings of the SC-SFM heterostructure for either the magnetic field $\mathbf{H}$ or the vector potential $\mathbf{A}$, amongst other PDE models ${ }^{48}$, are doomed to find the same local solution at the SC domain, as it still represents the simplest and mathematically valid solution which simply neglects any possible magnetostatic coupling between the SC and the SFM unless it would have been explicitly included.

Nevertheless, inspired by the pioneer research of Arnaud Devred at CERN on the determination of the magnetic field produced by accelerator magnets ${ }^{49-52}$, and the variational principles of the critical state theory originally introduced by A. Badía and C. Lópe $z^{53,54}$, which have been extensively used to demonstrate the occurrence of intriguing phenomena in type-II SC rounded wires by Ruiz et. al. ${ }^{10,55-61}$, in this paper we extend the integral formulation of the critical state theory introduced in Ref. 45, by including a multipole fields expansion which enables the straightforward inclusion of the magnetostatic coupling between a SC and a rounded SFM sheath (Sec. 2), explaining by semi-analytical and numerical methods the causes behind the increment of the AC-losses in a SC-SFM cylindrical metastructure (Sec. 3), and how it is affected by the amplitude of the current and the magnitude of the relative magnetic permeability of the SFM, as well as the main conclusions of this study (Sec. 4).

\section{Multipole Expansion of the Critical State Model in SC-SFM Rounded Heterostructures}

The fundamentals of the variational theory for the electromagnetic modeling of type II superconductors ${ }^{53,54}$, and therefore SC-SFM heterostructures, roots into the application of the optimal control theory for the minimization of the electromagnetic Lagrangian, $\operatorname{Min}\{\mathscr{L}\} \equiv \operatorname{Max}\{\mathbf{J} \cdot \mathbf{p}\}$, which is equivalent to the maximum projection rule of the power density with the electromagnetic Lagrangian multiplier defined as $\mathbf{p}=-\Delta \mathbf{A}=\mathbf{E} \Delta t$, for arbitrary variations of the magnetic field $\Delta \mathbf{B}=-\nabla \times \mathbf{p}^{45}$. In this way, a small linear path-step between two successive profiles of magnetic field, $\Delta \mathbf{B}=\mathbf{B}_{n+1}-\mathbf{B}_{n}$, fulfils Ampère's law $\nabla \times \mathbf{B}_{n}=\mu_{0} \mathbf{J}_{n}$, as well as $\nabla \cdot \mathbf{B}_{n}=0$ and $\nabla \cdot \mathbf{J}_{n}=0$, by imposing the minimization of the step variation for the magnetic field profile integral across the whole $\mathfrak{R}^{3}$-space,

$$
\mathscr{F}[\mathbf{B}(\cdot)]=\operatorname{Min} \int_{\mathfrak{R}^{3}} \frac{1}{2}|\Delta \mathbf{B}|^{2},
$$

where the SC domain, $\Omega_{S C}$, is conditioned to the $\mathbf{E}-\mathbf{J}$ material law which in the SC critical state model can be simplified to the inequality constraint $\mathbf{J} \leq \mathbf{J}_{c}$, and the transport current condition (if any),

$$
\int_{S C} \mathbf{J} \cdot \hat{\mathbf{n}} d \Omega=\mathbf{I}_{t r} .
$$




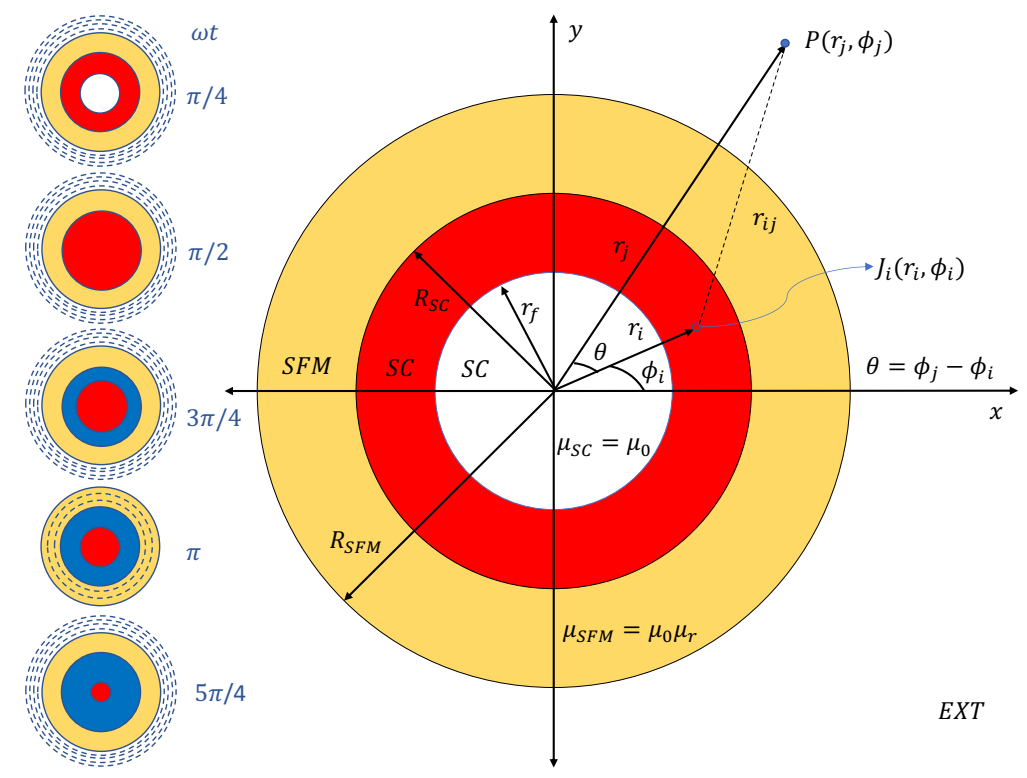

Figure 1. Pictorial representation of the cylindrical Superconducting (SC) - Soft-Ferromagnetic (SFM) metastructure analysed in this paper. The main plot shows the distribution of current density inside the SC under self-field conditions for an applied transport current $I_{t r}=I_{c} \sin (\omega t)$, with $\omega t=\pi / 4$ (red shadowed area), and the relative coordinates for a finite element of current density $J_{i}\left(r_{i}, \phi_{i}\right)$ as reference for the calculations in Eq. 5 to Eq. 18. The column of plots at the left illustrate the time dynamics of the current density and flux front profile inside the SC, together with the contour lines of magnetic field at the exterior (EXT) of the SC-SFM metastructure, for the sake of completeness.

Then, for a 2D system as the one considered in Fig. 1, where the elements of current density can only flow along the $z$-axis and therefore, where the dynamics of flux front profiles is restricted to the $x-y$ plane, the minimization functional can be simply rewritten as,

$$
\mathscr{F}[\mathbf{A}(\cdot)]=\operatorname{Min} \int_{\mathfrak{R}^{2}}\left[\Delta \mathbf{A}_{z} \cdot \mathbf{J}+\nabla \phi \cdot \mathbf{J} \Delta t\right],
$$

where the gradient of the scalar electric potential $\nabla \phi=C_{t} \hat{u}_{z}$ is different to zero only if $I_{t r} \neq 0$, with the electric field and magnetic vector potential directed along the $z-$ axis, $\mathbf{E}_{z}=-\partial_{t} \mathbf{A}+C_{t}$. Thus, as the AC-losses of the system are determined by the integral of the instantaneous power density losses across the material domains, $\Omega$, over a hysteresis cycle of the transport current excitation of frequency $\omega$, i.e.,

$$
L=\omega \oint_{t} \int_{\Omega} \mathbf{E} \cdot \mathbf{J} d \Omega d t
$$

then, the problem simply reduces to determine the magnetic vector potential across the different material domains with current density. Thus, in a first approach it would seem impossible to predict a rise in the AC-losses of SC-SFM metastructures under self field conditions, as not only induced magnetic losses by the non-hysteretic SFM can be neglected ${ }^{12,35}$, but also no current sharing is to be seen between the SC and the SFM material ${ }^{62}$. Therefore, with no magnetization losses neither current profiles within the SFM and, exactly the same distribution of current density inside the SC domain which for a cylindrical wire of radius $R_{S C}$ can be determined by exact analytical methods ${ }^{47}$, i.e., as the area enclosed between its surface and the circumference of radius

$$
r_{f}=R_{S C} \sqrt{1-\frac{I_{t r}}{I_{c}}}
$$

being $r_{f}$ the inner boundary of the flux front profile, then any possible change in the losses of the system will be restricted to the definition of the electric field invoked into the SC domain.

This immediately allow us to identify why conventional PDE solvers such as COMSOL Multiphysics cannot predict the increment in the AC losses in the SC domain, as the material law that governs the physics of the macroscopic magnetic behavior of a SC is directly entered by the empirical ansatz known as the $\mathbf{E}-\mathbf{J}$ power law. This although it is a very acknowledged and 
successful way to reproduce the electromagnetic behavior of practical type II superconductors and many of their applications, it also forces the electric field to be a known function primarily measured at the self-field critical current density condition, which commonly does not take into consideration any variance into the magnetic vector potential (A) nor any possible contribution by other materials such as a SFM. However, within the integral formulation of Eq. 1, and consequently Eq. 2, the electric field is seldom calculated by the use of empirical material laws for the SC state, but instead from the well established Bean's theorem for the critical state theory which reads as $\mathbf{J} \leq \mathbf{J}_{c}{ }^{53,63}$, as well as from the knowledge of an analytical function for defining the magnetic vector potential along the $\mathfrak{R}^{3}$ space. This in turn can be transformed into a function of finite elements of current density $\mathbf{J}_{i}$ that multiplied by their inductance matrices, which do not depend on any physical variable but on the position of the elements of current $\left(\mathbf{r}_{i}\right)$, generally leads to a reduction on the size and dimensionality of the minimization integral from the whole $\Re^{3}$-space ${ }^{45,56,64}$, to just the volume or area of the SC domain $\Omega_{S C}$.

Therefore, if in the 2D geometry shown in Fig. 1 we assume that the elements of current density appear only inside of the SC domain by the injection of transport current, and these can be treated as infinitely long and thin wires, then the vector potential for the self and mutual inductances in the absence of the SFM can be written as

$$
\begin{aligned}
& \mathbf{A}_{i}\left(\mathbf{r}_{i}\right)=\left(\mu_{0} / 4 \pi\right) \pi \mathbf{J}_{i}, \\
& \mathbf{A}_{i j}\left(\mathbf{r}_{j}\right)=-\left(\mu_{0} / 4 \pi\right) \ln \left(r_{i j}^{2}\right) \mathbf{J}_{i} \forall r_{i j} \neq 0,
\end{aligned}
$$

with $r_{i j}$ the distance between two lines of current each at the positions $r_{i}$ and $r_{j}$. Then, in order to formulate the magnetic vector potential in the case of a SC-SFM metastructure, the starting point is to define the distance $r_{i j}$ in the complex or $s-p l a n e$ as $r_{i j}=r_{i}-s$, with $s=r_{j} e^{i \theta}$ (see Fig. 1), such that $\operatorname{Re}\{\mathbf{A}\}=A_{z}$, with the vector potential created by a line of current $\mathbf{J}_{i}$ at any position $\mathbf{r}_{j} \neq \mathbf{r}_{i}$, being defined by

$$
\mathbf{A}_{i j}\left(\mathbf{r}_{j}\right)=-\frac{\mu_{0}}{2 \pi} \mathbf{J}_{i} \ln \left(\kappa r_{i j}\right) \forall r_{j} \in \kappa
$$

Here the index $\kappa= \pm 1$ separates the space into the conditions $0<r_{j}<r_{i}$ for $\kappa=1$, a condition that is useful for beam optics computations in the case of accelerator magnets ${ }^{49}$, and the condition $r_{j}>r_{i}$ with $\kappa=-1$ which results useful for magnetic computations $^{50}$. Then, the key instrument is to expand the function $\ln \left(r_{i j}\right)=\ln \left(r_{i}\right)+\ln \left(1-s / r_{i}\right)$ into a Taylor's series that with the help of De Moivre's Formula allows to rewrite Eq. 8 as:

$$
\mathbf{A}_{i, j}=-\frac{\mu_{0}}{2 \pi} \mathbf{J}_{i}\left[\ln \left(r_{k}\right)-\sum_{n=1}^{\infty} \frac{1}{n}\left(\frac{r_{j}}{r_{i}}\right)^{\kappa n} \cos (n \theta)\right],
$$

with $r_{k}=r_{i}$ for $\kappa=1$, and $r_{k}=r_{j}$ for $\kappa=-1$, where it is to be noted that the vector potential is continuous at $r_{j}=r_{i}$.

Then, when considering the SFM medium the problem can be solved by Laplace's equation $\square^{2} \mathbf{A}=\nabla^{2} \mathbf{A}-\partial_{t}^{2} \mathbf{A} / c^{2}=$ $-4 \pi c^{-1} \mu_{r} \mathbf{J}$, which in the magneto quasi-steady approach introduced in Ref. 45 , i.e., with $\partial_{t}^{2} \mathbf{A}=0$, can be simplified to $c \nabla^{2} \mathbf{A}=0$, as no current sharing can be assumed between the SC and the SFM. Thus, in the case of a SC-SFM metastructure as the one shown in Fig. 1, this equation can be solved in cylindrical coordinates by the method of separation of variables, such that its solution can be expressed as $A_{c, m}=R(r) \Theta(\theta)$ with $\Theta(\theta)$ a $2 \pi$ periodic function of $\theta$, and the Laplace' equation simplified to

$$
\frac{r}{R} \frac{\partial}{\partial r}\left(r \frac{\partial R}{\partial r}\right)=-\frac{1}{\Theta} \frac{\partial^{2} \Theta}{\partial \theta^{2}}=C,
$$

with $C$ a real constant that do not depend on $r$ nor $\theta$.

Consequently, the most general solution to Eq. 10 is a linear superposition for all possible solutions ${ }^{52}$ either with $C=0$, $C>0$, or $C<0$, resulting in the general definition for the vector potential in the absence of current density for a coupled medium $m$,

$$
\begin{aligned}
\mathbf{A}_{c, m}=\quad & E_{0, m} C_{0, m}+D_{0, m} \ln (r) \\
& +\sum_{n=1}^{\infty} E_{n, m} \cos (n \theta)\left(C_{n, m} r^{n}+D_{n, m} r^{-n}\right),
\end{aligned}
$$

with the unknown media-dependent parameters $C_{m}, D_{m}$, and $E_{m}$ being real integration constants which can be determined by superimposing the vector potential created by the mere existence of a media, i.e., Eq. 11, with the one created by the existence of a line of current, i.e., Eq. 9, and also by further imposing adequate boundary conditions at the interfaces between the different media. 
Thus, for the SC-SFM metastructure shown in Fig. 1, the magnetic vector potential created by a line of current $J_{i}$ located at $r_{i}$ over a point in the space $r_{j} \neq r_{i}$, i.e., $A_{z, m}\left(r_{j}\right)=A_{i, j}\left(r_{k}\right)+A_{m}\left(r_{j}\right)$, must be defined within four different regions of the space, two of these within the SC domain for the conditions $\kappa=1$ (i.e., $0<r_{j}<r_{i}$ ) and $\kappa=-1$ (i.e., $r_{i}<r_{j}<R_{S C}$ ), and the other two defining the space occupied by the SFM layer $\left(R_{S C}<r_{j}<R_{S F M}\right)$, and the outer domain (EXT) defined by the condition $R_{S F M}<r_{j}$ (see Fig. 1). Then, in order to get an unequivocal physical solution, this system of equations must satisfy continuity boundary conditions at $r_{j}=R_{S C}$ and $r=R_{S F M}$, i.e. at the interfaces between two different mediums, both for the magnetic vector potential $A_{z}$, i.e, by the conditions $A_{z, S C}\left(R_{S C}\right)=A_{z, S F M}\left(R_{S C}\right)$ and $A_{z, S F M}\left(R_{S F M}\right)=A_{z, E X T}\left(R_{S F M}\right)$, as well as for the magnetic field vector $\mathbf{B}=\nabla \times \mathbf{A}_{z}$, such that the condition of non divergence of the magnetic field is preserved by satisfying the conditions $\partial_{r} A_{z, S C}=\mu_{r}^{-1} \partial_{r} A_{z, S F M}$ and $\partial_{r} A_{z, E X T}=\mu_{r}^{-1} \partial_{r} A_{z, S F M}$ for $r=R_{S C}$ and $r=R_{S F M}$, respectively.

This creates a set of minimum six equations at each one of the interfaces between the different media, from which we can determine the set of six media-dependent constants $C_{0, m}, C_{n, m}, D_{0, m}, D_{n, m}, E_{0, m}$, and $E_{n, m}$. Thus, after some algebra it is possible to demonstrate that the total vector potential for the different media, SC, SFM, and EXT in Fig. 1 can be written as:

$$
\begin{aligned}
& \mathbf{A}_{S C}\left(\mathbf{r}_{j} \leq R_{S C}\right)=\mathbf{A}_{j}\left(\mathbf{r}_{j}\right)+\sum_{i \neq j} \mathbf{A}_{i j}\left(\mathbf{r}_{j}\right)+\mathbf{A}_{c, S C}\left(\mathbf{r}_{j}\right), \\
& \mathbf{A}_{S F M}\left(R_{S C}<\mathbf{r}_{j} \leq R_{S F M}\right)=\sum_{i} \mathbf{A}_{i j}\left(\mathbf{r}_{j}\right)+\mathbf{A}_{c, S F M}\left(\mathbf{r}_{j}\right), \\
& \mathbf{A}_{E X T}\left(R_{S F M}<\mathbf{r}_{j}\right)=\sum_{i} \mathbf{A}_{i j}\left(\mathbf{r}_{j}\right)+\mathbf{A}_{c, E X T}\left(\mathbf{r}_{j}\right),
\end{aligned}
$$

with the vector potentials for the coupled media defined by,

$$
\begin{aligned}
& \mathbf{A}_{c, S C}=-\frac{\mu_{0}}{2 \pi} \mu_{(-)} \mathbf{J}_{i}\left[\mu_{(+)} \sum_{n=1}^{\infty} \frac{\bar{R}_{\mu 1}}{n}\left(\frac{r_{i} r_{j}}{R_{S C}^{2}}\right)^{n} \cos \left(n \phi_{j}\right)\right] ; \text { with } \bar{R}_{\mu 1}=\frac{R_{S F M}^{2 n}-R_{S C}^{2 n}}{\mu_{(-)}^{2} R_{S C}^{2 n}-\mu_{(+)}^{2} R_{S F M}^{2 n}}, \\
& \mathbf{A}_{c, S F M}=\frac{\mu_{0} \mu_{(-)}}{2 \pi} \mathbf{J}_{i}\left[\ln \left(\frac{R_{S C}}{r_{j}}\right)-\sum_{n=1}^{\infty} \frac{\bar{R}_{\mu 2-}}{n}\left(\frac{r_{i}}{r_{j}}\right)^{n} \cos \left(n \phi_{j}\right)\right] ; \bar{R}_{\mu 2 \pm}=\frac{\mu_{(+)} R_{S F M}^{2 n} \pm 2 \mu_{r} r_{j}^{2 n}+\mu_{(-)} R_{S C}^{2 n}}{\mu_{(-)}^{2} R_{S C}^{2 n}-\mu_{(+)}^{2} R_{S F M}^{2 n}} \\
& \mathbf{A}_{c, E X T}=\frac{\mu_{0}}{2 \pi} \mu_{(-)} \mathbf{J}_{i}\left[\ln \left(\frac{R_{S C}}{R_{S F M}}\right)+\mu_{(-)} \sum_{n=1}^{\infty} \frac{\bar{R}_{\mu 1}}{n}\left(\frac{r_{i}}{r_{j}}\right)^{n} \cos \left(n \phi_{j}\right)\right]
\end{aligned}
$$

with $\mu_{( \pm)}=\mu_{r} \pm 1$, such that if the magnetic properties of the SFM are removed, i.e., if its magnetic permeability takes the value of the relative magnetic permeability of vacuum $\mu_{r}=1$, then all the coupling contributions in Eq. 12 disappear, returning to the classical problem where the distribution of current into a superconductor can be calculated by the simple knowledge of the self and inductance matrices for finite elements of critical current density ${ }^{56}$.

Likewise, we have introduced the non-dimensional factors $\bar{R}_{\mu 1}$ and $\bar{R}_{\mu 2 \pm}$, where it is to be noticed that $\bar{R}_{\mu 2 \pm}$ is not a constant but a function of the element coordinate $r_{j}$. Also, it is to be noticed that these contributions are a response of the SFM to the lines of current density $\mathbf{J}_{i}$ inside the SC, which are calculated through the minimization functional shown in Eq. 2. Therefore, within the integral formulation in Eq. 3, and the vector potentials obtained in Eqs. 12-15, the system is reduced to the calculation of the profiles of current density inside the SC only, providing a tremendous advantage against any other method because not only the coupling between the SC and SFM are explicitly included, but also because the infinite $\mathfrak{R}^{2}$-space has been reduced to just the area occupied by the SC domain. Additionally, although the coupling terms depend on the reach of the n-index for the introduced Taylor's series, it is to be noticed that the arguments of these summations are purely geometrical, therefore defining the multipole coefficients for the coupling inductance matrices between the SC and the SFM. Thus, for a given finite element mesh these can be univocally calculated outside of the minimization process, with the resulting matrix for the positions $r_{i}$ and $r_{j}$ being stored as a matrix of constant parameters into the minimization algorithm, thence, substantially reducing its computing time.

Therefore, the only limitation of this method lies in the computational limits and numerical precision for the calculation of the multipole coefficients for the coupled-media vector potentials, which refers to the smallest and the largest positive normalized floating-point number in IEEE ${ }^{\circledR}$ double precision, i.e., $2^{-1022}$ and $\left(2-2^{-52}\right) * 2^{1023}$, respectively. In this sense, if the SC and SFM radii are written in normalized units such that, $R_{S C}=1$ and $R_{S F M}=1.5 R_{S C}$, for the effects of the minimization process of the functional of interest, $\mathscr{F}[\mathbf{A}(\cdot)]=\mathscr{F}_{S C}\left[\mathbf{A}_{S C}\left(\mathbf{J}_{i}\right), C_{t}\right]$, the largest n-index that could be considered is $n=\log \left(1.7977 \times 10^{308}\right) / \log (1.5) \simeq 1750$, from which we have found that within a $10^{-8}$ tolerance factor, any $\mathrm{n}$-index greater than $\sim 350$ will produce the same results. Then, by knowing the total magnetic vector potential across the whole space, it is possible to numerically determine the distribution of current density $\mathbf{J}_{i}$ inside the SC-SFM metastructure for a given time, by 
solving the minimization functional $\mathscr{F}\left[\mathbf{A}_{S C}\left(\mathbf{J}_{i}\right), C_{t}\right]$ subject to the critical state condition $\left|J_{i}\right| \leq J_{c}$, and the applied transport current constraint, $I_{t r}(t)=I_{0} \sin (\omega t)$ in Eq. 2, with $I_{0}$ the amplitude of the alternating current (AC) of frequency $\omega$.

It is worth reminding that the constant $C_{t}$ into the minimization fucntional must be introduced into the numerical procedure in order to determine correct value for the electric field and consequently for the AC losses, such that the electric field at flux free regions satisfy the condition $E_{z}\left(r_{j}<r_{f}\right) \equiv 0^{56}$. Nevertheless, only very small increments in the magnitude of the electric field inside the SC have been observed by the coupling with the SFM sheath, these in the order of $1 \times 10^{-3}\left(\mu_{0} / 4 \pi\right) R_{S C}^{2} J_{c} \delta t^{-1}$, such that the local distribution of power density $\mathbf{E} \cdot \mathbf{J}$ shows not only the same classical behaviour already shown for bare SC wires $^{57,65}$, but exactly the same distribution of local profiles of critical current density that could be calculated by analytical methods, simultaneously proving the accurateness of our minimization procedure. Thus, although the slight increment in the time-dependent electric field inside the SC ultimately contributes to the increment of the hysteresis losses of the SC-SFM system, it does not provide a very rich physics phenomenology which could reveal the actual impact of the SFM coupling with the SC current, or at least not beyond a prospective increment in the AC-losses of the system which fundamentally depends on the relative magnetic permeability of the SFM. Nevertheless, in our attempt to fully answer the question, How the relative magnetic permeability of a SFM sheath affects the AC-losses of a SC wire? we have conducted a large number of simulations (330), including 10 different amplitudes of $I_{0}$, ranging from $0.1 I_{c}$ to $I_{c}$, for at least 33 different SFM with relative magnetic permeabilities that range from, $\mu_{r}=5$ for NiZn ferrites ${ }^{66}$, up to the very high magnetic permeability found for purest Iron $\mu_{r}=350000^{66-68}$, what has allowed us to unveil that the key fingerprint or most distinctive and notorious feature of the SFM-SC coupling lies in the distribution of local profiles of magnetic field, it due to the induced magnetic multipoles created by the interaction between the supercurrents and the SFM sheath.

Thus, either by calculating the distribution of profiles of current density by the minimization functional $\mathscr{F}\left[\mathbf{A}_{S C}\left(\mathbf{J}_{i}\right)\right]$ or, by directly meshing the distribution of profiles of current density $J_{i}$ within the analytically derived flux front boundary $r_{f}$ (Eq. 5), the magnetic field can be calculated by its general definition $\mathbf{B}=\nabla \times \mathbf{A}$, which for our 2D cylindrical geometry (see Fig. 1) is reduced to $\mathbf{B}=r^{-1} \partial_{\phi} A_{z} \hat{\mathbf{u}}_{r}-\partial_{r} A_{z} \hat{\mathbf{u}}_{\phi}$, where $A_{z}$ is split into the three continuous media $A_{S C}, A_{S F M}$, and $A_{E X T}$ at Eq. 12. Therefore, by calculating the corresponding derivatives, we have obtained that at each one of the domains representing these media, the components of the magnetic field can be calculated by the functions,

$$
\begin{gathered}
\mathbf{B}_{S C}=\frac{\mu_{0}}{2 \pi} J_{i}\left\{\begin{array}{l}
{\left[\frac{r_{i}}{r_{i j}^{2}} \sin \left(\phi_{i}-\phi_{j}\right)+\mu_{(-)} \mu_{(+)} \sum_{n=1}^{\infty} \frac{\bar{R}_{\mu 1}}{r_{j}}\left(\frac{r_{i} r_{j}}{R_{S C}^{2}}\right)^{n} \sin \left(n \phi_{j}\right)\right] \hat{\mathbf{u}}_{r}} \\
{\left[\frac{r_{j}}{r_{i j}^{2}}-\frac{r_{i}}{r_{i j}^{2}} \cos \left(\phi_{i}-\phi_{j}\right)+\mu_{(-)} \mu_{(+)} \sum_{n=1}^{\infty} \frac{\bar{R}_{\mu 1}}{r_{j}}\left(\frac{r_{i} r_{j}}{R_{S C}^{2}}\right)^{n} \cos \left(n \phi_{j}\right)\right] \hat{\mathbf{u}}_{\phi}}
\end{array}\right\}, \\
\mathbf{B}_{S F M}=\frac{\mu_{0}}{2 \pi} J_{i}\left\{\begin{array}{l}
{\left[\frac{r_{i}}{r_{i j}^{2}} \sin \left(\phi_{i}-\phi_{j}\right)+\mu_{(-)} \sum_{n=1}^{\infty} \frac{\bar{R}_{\mu 2-}}{r_{j}}\left(\frac{r_{i}}{r_{j}}\right)^{n} \sin \left(n \phi_{j}\right)\right] \hat{\mathbf{u}}_{r}} \\
{\left[\frac{r_{j}}{r_{i j}^{2}}-\frac{r_{i}}{r_{i j}^{2}} \cos \left(\phi_{i}-\phi_{j}\right)+\mu_{(-)} \sum_{n=1}^{\infty}\left[1-\left(\frac{r_{i}}{r_{j}}\right)^{n} \bar{R}_{\mu 2+}\right]\left(\frac{1}{r_{j}}\right) \cos \left(n \phi_{j}\right)\right] \hat{\mathbf{u}}_{\phi}}
\end{array}\right\}, \\
\mathbf{B}_{E X T}=\frac{\mu_{0}}{2 \pi} J_{i}\left\{\begin{array}{l}
{\left[\frac{r_{i}}{r_{i j}^{2}} \sin \left(\phi_{i}-\phi_{j}\right)-\mu_{(-)}^{2} \sum_{n=1}^{\infty} \frac{\bar{R}_{\mu 1}}{r_{j}}\left(\frac{r_{i}}{r_{j}}\right)^{n} \sin \left(n \phi_{j}\right)\right] \hat{\mathbf{u}}_{r}} \\
{\left[\frac{r_{j}}{r_{i j}^{2}}-\frac{r_{i}}{r_{i j}^{2}} \cos \left(\phi_{i}-\phi_{j}\right)+\mu_{(-)}^{2} \sum_{n=1}^{\infty} \frac{\bar{R}_{\mu 1}}{r_{j}}\left(\frac{r_{i}}{r_{j}}\right)^{n} \cos \left(n \phi_{j}\right)\right] \hat{\mathbf{u}}_{\phi}}
\end{array}\right\} .
\end{gathered}
$$

In this way, we have found analytical functions for the magnetic field than can be used with the distribution of profiles of current density calculated by the numerical minimization of the optimal control functional in Eq. 3, which as mentioned above has resulted to be no other than the one that could be calculated by means of the flux-front profile defined by Eq. 5. In this sense, we have arrived to a fully analytical solution for the magnetic vector potential and the distribution of the magnetic field inside the SC core of a cylindrical SC-SFM metastructure, it subjected to an AC transport current and under self-field conditions, allowing to demonstrate the physics origin of the deformations of the magnetic field inside the SC observed by magneto optical imaging techniques ${ }^{39,41,43,69}$, and the intriguing increment in the AC-losses as result of the magneto-steady coupling between the SC and the SFM sheath ${ }^{36-38}$. 

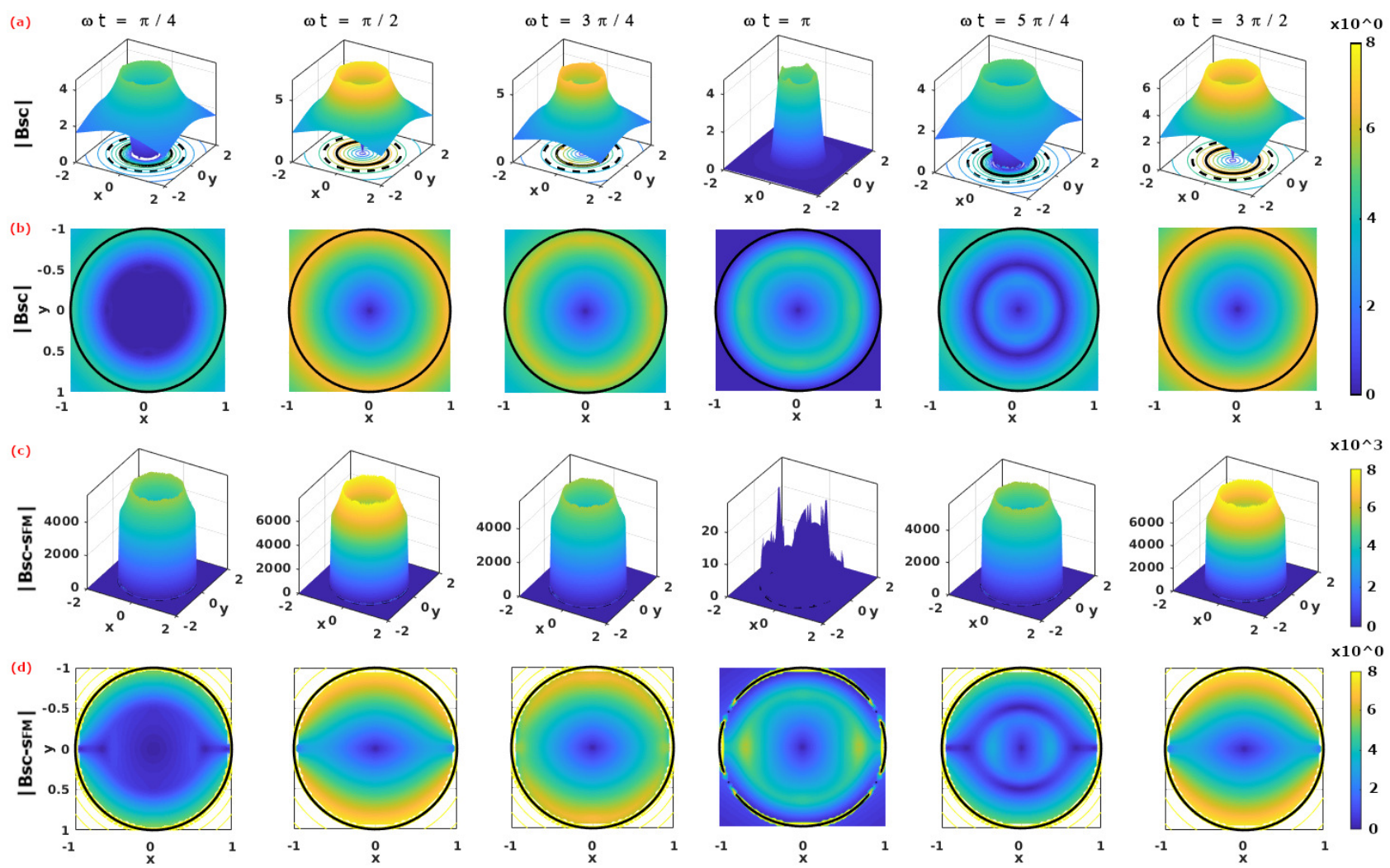

Figure 2. Dynamics of the norm of magnetic flux density $|\mathbf{B}|$ in units of $\left(\mu_{0} / 4 \pi\right) J_{c} R_{S C}$ in (a) a SC wire of radius $R_{S C}=1$ (in arbitrary units) whose cross section lies on the plane $x y$ and which is subjected to an applied transport current $I_{t r}=I_{C} \sin (\omega t)$. (b) shows the same distribution of field but in a 2D representation that focus on the local flux dynamics inside the SC, $\left|\mathbf{B}_{S C}\right|$. Analogously, (c) shows the flux distribution $\left|\mathbf{B}_{S C-S F M}\right|$ for the SC-SFM metastructure with $R_{S F M}=1.5 R_{S C}$ and $\mu_{r}=46$, where the impact of the SFM on the SC can be seen clearer in the 2D representation shown in (d), i.e., the bottom pane of subplots. The time interval between columns is $\Delta t=(\pi / 4) \omega^{-1}$, such that the dynamics shown between the second column $(\omega t=3 * \pi / 2)$ and last column $(\omega t=\pi / 2)$ represents the minimum hysteresis period for the calculation of AC Losses, in accordance with the distribution of profiles of current density shown in Fig. 1.

\section{Differences on the Local Magnetic Field Distributions and AC-Losses of SC-SFM Het- erostructures}

In Fig. 2, the norm of the magnetic field is shown as a function of the non-dimensional time argument $\omega t$ of the applied transport current, $I_{t r}=I_{0} \sin (\omega t)$, with maximum amplitude, $I_{0}=I_{c}$, illustrating its behaviour during the first ramp of the AC current at $\omega t=\pi / 4$ (1st column), as well as during the hysteretic period observed between the peaks $\omega t=2 \pi$ ( 2 nd column) and $\omega t=3 \pi / 2$ (6th column), as it suffices for the calculation of the AC-losses when the time integral in Eq. 4 is defined between these time-steps and then, multiplied by a factor 2 .

For the sake of comparison, the results presented are shown under two different considerations: (i) the first (top two rows) refers to the case when $\mu_{r}=1$, i.e., in absence of the SFM sheath, and (ii) the second (bottom two rows) makes reference to the case where the SFM sheath in Fig. 1 is defined by a relative radius $R_{S F M}=1.5 R_{S C}$ and a magnetic permeability $\mu_{r}=46$, being this a typical magnetic permeability encountered for $\mathrm{MgB}_{2}-\mathrm{Fe}$ wires ${ }^{12,34,39,69}$. Thus, it is to be noticed that as consequence of the magnetic coupling with the SFM sheath, a remarkable deformation of the local density of magnetic flux inside the SC has been found (bottom pane in Fig. 2), despite that fact that the distribution of current density still follows the circumferential evolution of current density profiles observed for unsheathed SC wires, i.e., delimited by the flux front analytically derived in Eq. 5, and which has been shown in Fig. 1 for illustration purposes. Notoriously, this observation is in remarkable agreement with the the experimental evidences for the local distribution of magnetic field inside SFM sheathed SC monowires reported by magneto optical imaging techniques ${ }^{39,41,43,69}$, being this the first model in the literature capable reproduce this behaviour within the well acknowledged critical state regime. 

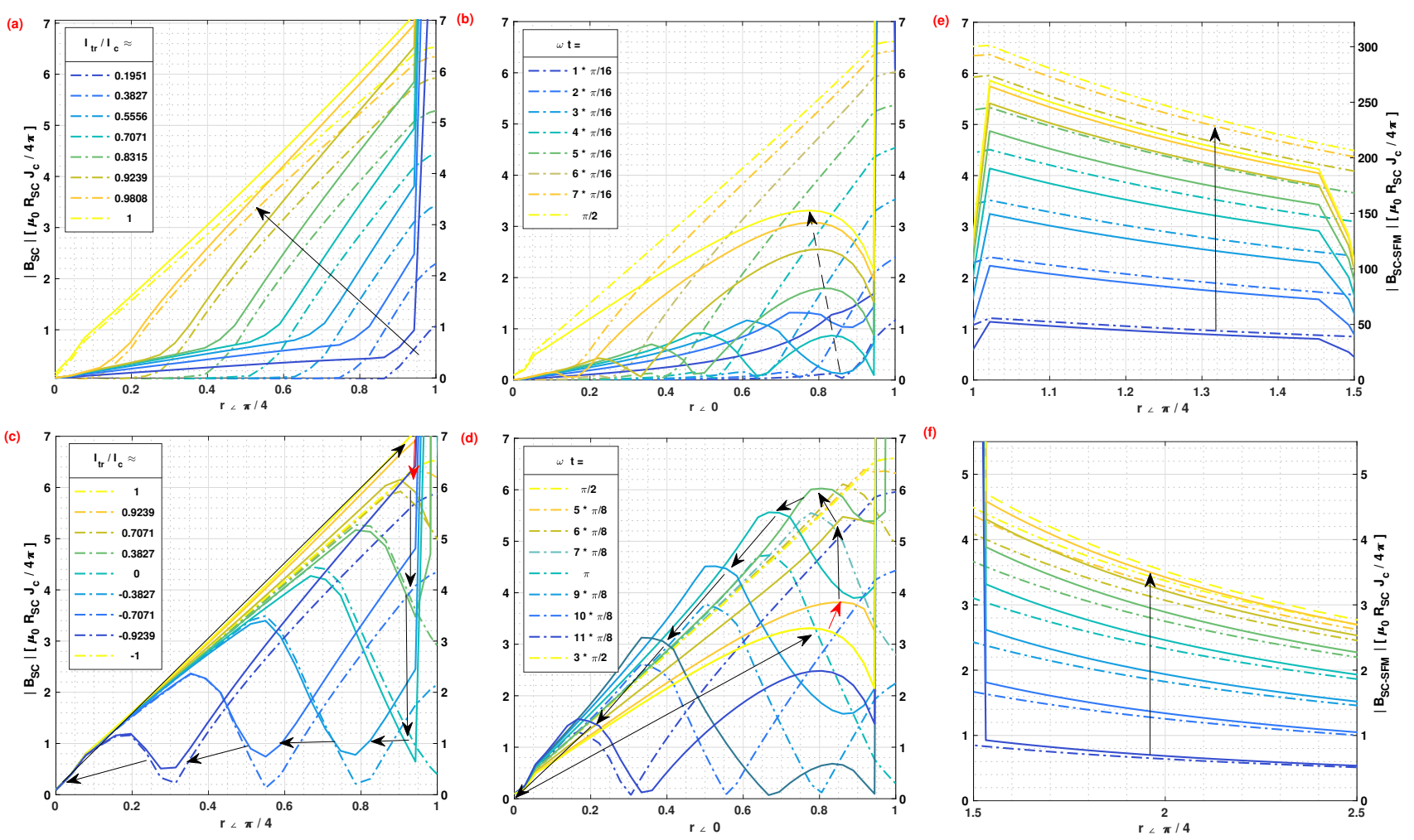

Figure 3. Dynamics of the norm of magnetic flux density over the radial directions $(r, \pi / 4)$ and $(r, 0)$ for different magnitudes of the applied AC transport current, $I_{t r}=I_{c} \sin (\omega t)$, it measured inside the SC during (a-b) the first ramp of the applied current and (c-d) the peak-to-peak hysteretic period as described in Figs. 1 \& 2. Left pane of subplots show the corresponding profiles for the first ramp of current at (e) inside the SFM and (f) outside the SC-SFM wire, respectively. Dashed-dot lines at each subplot refer to the left axis $\left|\mathbf{B}_{S C}\right|$ showing a classical Bean's behaviour, whilst the solid lines must be read accordingly with the right hand axis $\left|\mathbf{B}_{S C-S F M}\right|$. The arrows show the 'time' evolution of the field profiles, and units for $\mathbf{B}$ are $\left(\mu_{0} / 4 \pi\right) J_{C} R_{S C}$.

Within these experiments a characteristic "elevation" and "dip" of the magnetic flux at self-field conditions near the SC-SFM interface at the line-angle $r \angle 0$ has been observed, i.e., at the $x$-axis from the observer's perspective. Without the prove here presented, this phenomena could be thought to be in apparent contradiction with the critical state regime, as not only some magnetic field appeared at the so-called flux-free regions, i.e, regions where no current density is expected to be flowing, but also, because it does not have a qualitative resemblance with the angular invariant pattern that can be observed for the magnetic field at the exterior of the SC wire, regardless whether the SC wire is sheathed by a SFM or not. Thus, this intriguing phenomena was originally thought to be caused by some mechanism similar to the overcritical state model of Genenko et. al. for thin strips $^{23,25,26}$, although in the case of rounded SC-SFM wires it could not be validated, it because these so-called overcritical currents have not been ever reported by direct experimental measurements ${ }^{18,41,43,44}$. Nevertheless, we have demonstrated that the inclusion of magnetic multipoles by the physical coupling between the SC and the SFM, are sufficient to reproduce all the macroscopic electromagnetic features of SC-SFM rounded metastructures, without violating the most fundamental principles of the critical state theory for type II superconductors ${ }^{45}$.

All the above can be seen in better detail from Fig. 3, where we have displayed the local profiles for the norm of the magnetic field along two different radial directions, i.e., along the $(r, \phi)$ lines with $\phi=0$ and $\phi=\pi / 4$, respectively, either (i) inside the SC wire $(0<r<1)$, (ii) inside the SFM $(1<r<1.5)$, or (iii) outside the SC-SFM metastructure $(1.5<r)$. The magnetic behavior of the SC-SFM (solid lines) is compared with the classical critical-state behavior computed for an unsheathed SC wire (dashed lines), where besides the rapid rise of the magnetic field at the interface between the SC and the SFM, no disturbance of the magnetic field has been observed along the $y$-axis $(\phi=\pi / 2)$, in good agreement with the experimental measurements for $\mathrm{Fe}\left(\mu_{r}=46\right)$ sheathed $\mathrm{MgB}_{2}$ wires $39,41,43,69$, which reported the unusual "elevation" and "dip" of the magnetic flux only around the interface between the SC and the SFM when $\phi=0$ (or $\pi$ ). In fact, the non-divergence and continuity conditions of the magnetic field can be directly observed in this figure, as the "elevation" in the flux free regions 


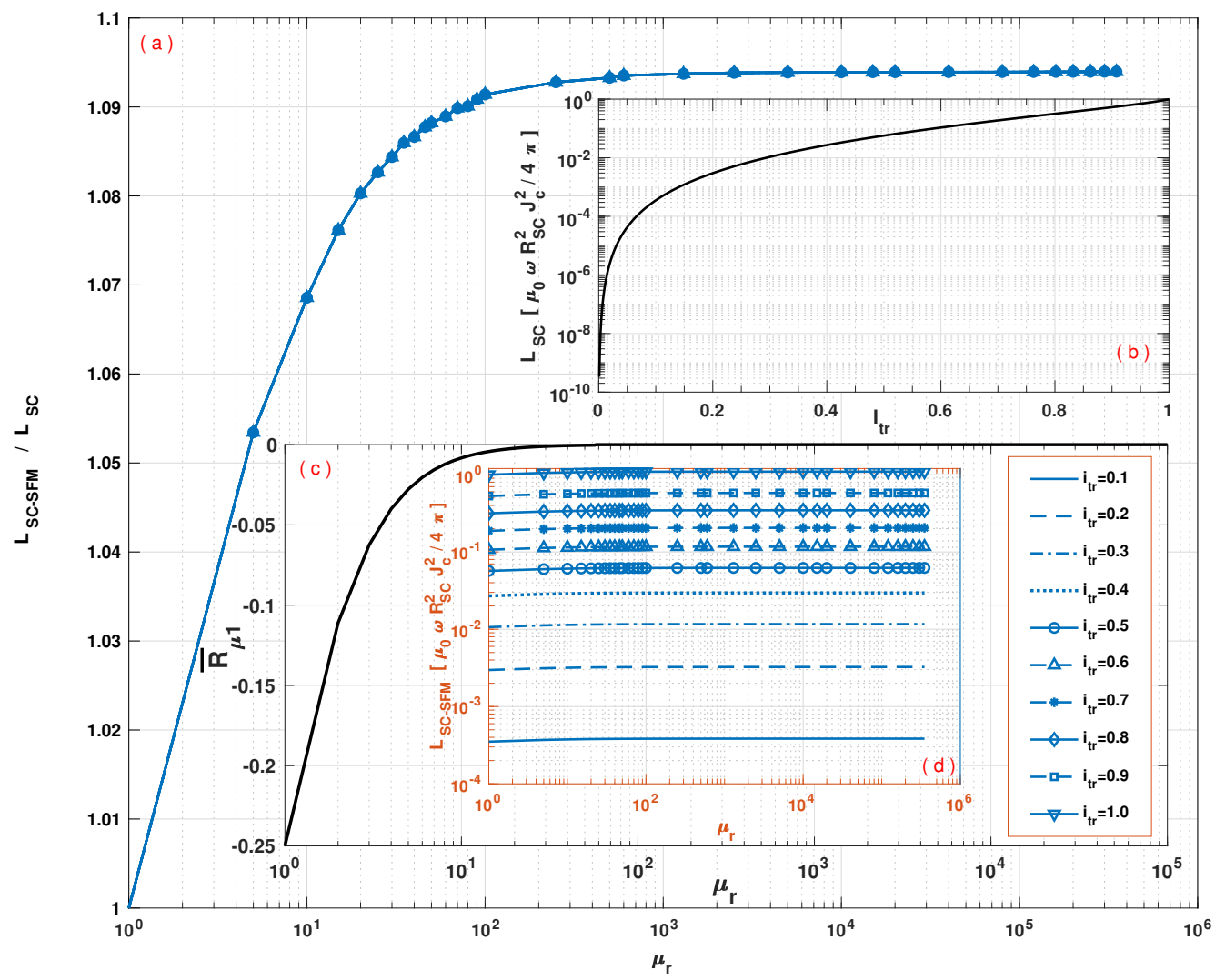

Figure 4. (a) Hysteretic losses ratio between SC-SFM metastructures $\left(L_{S C-S F M}\right)$ and the AC losses produced by an isolated SC wire $\left(L_{S C}\right)$ of cylindrical cross section as a function of the relative magnetic permeability $\mu_{r}$ of the SFM with

$R_{S F M}=1.5 R_{S C}$. (b) The top inset shows the well known analytical solution for $L_{S C}$ as a function of $I_{t r}{ }^{47,56,60}$. (c) The bottom outer inset shows the numerical tendency of the non-dimensional factor $\bar{R}_{\mu 1}$ at Eq. 13 valid for any radius of the SFM sheath up to $R_{S F M}=10 R_{S C}$ and with magnetic permeabilities from $\mu_{r} \sim 1$ up to $1 \times 10^{5}$. (d) Finally, the inner inset shows the dependence of the metastructure losses $\left(L_{S C-S F M}\right)$ for different amplitudes of the transport current $i_{t r}$ in units of $I_{c}$, and the relative magnetic permeability of the SFM sheath $\mu_{r}$.

develops symmetrically from the condition $\phi= \pm \pi / 2$ towards $\phi=0$, showing already a rise in the magnetic field along the line $(r, \phi / 4)$ inside the SC (Fig. 3 (a)) with the "dip" being evident at $\phi=0$ (Fig. 3 (b)), either from the first ramp of the applied transport current $(0<\omega t<\pi / 2)$, emulating the DC behavior, or during the hysteretic period shown in Figs. 3 (d)-(e) for the angles $\phi=0$ and $\phi=\pi / 4$, respectively.

Then, as it is shown in Fig. 3 (c) and Fig. 3 (e), besides the rapid change in the intensity of the magnetic field that occurs at the interfaces between the SC and the SFM at $r=1$, and the SFM and the EXT domain at $r=1.5$, which are both caused by the change in the relative magnetic permeability of the medium, there is almost a negligible change in the slope or pattern of the magnetic field profile outside the SC-SFM metastructure at self-field conditions, reaching nearly the same value of magnetic field between the bare SC wire and the SFM-sheated SC wire at a distance less than just twice the radius of the SC-SFM wire. Also, it is worth mentioning than the curves displayed in Fig. 3 refer directly to the calculations made within the numerical minimization framework of Eq. 3, i.e., with the profiles of current density directly calculated by our numerical method, and then used to calculate the magnetic field from our analytical derivations at Eqs. 16-18. Therefore, the exact position where the sudden rise or drop of the magnetic field near the interfaces abovementioned is shown, can be somehow overestimated as it depends on the size of the finite elements considered for defining the local profiles of current density $J_{i}$. Still, such features have been experimentally observed from magneto optical imaging measurements ${ }^{41,69}$, reason why for the purpose of proving the general validity of the critical state theory, we displayed here the numerically obtained results rather than the fully analytical ones, which could consider a much finer mesh by the knowledge of the distribution of profiles of current density from Eq. 5, under the expense of increasing the post-processing time with no further relevant physics. 
Finally, concerning to the energy losses of the SC-SFM wire, our model allows to prove that the sole coupling between the SC and the SFM sheath is capable to produce a slight rise in the AC-losses of the SC wire, even when the SFM layer does not add any electrical nor magnetic losses to the system (see Fig. 4). Likewise, it has been found how the selection of the SFM magnetic properties, i.e., its relative magnetic permeability, can affect the most important observable macroscopic quantities such as the magnetic field created by the SC-SFM metastructure, and its energy losses. To understand this, it is to be noticed than in Fig. 4, the curve of AC-losses for the SC-SFM metastructure, $L_{S C-S F M}$, has been calculated as a function of the relative magnetic permeability of the SFM, $\mu_{r}$, starting from the case of a bare SC, i.e., with $\mu_{r}=1$ (Fig. 4 (b)), up to an extremely high and rare magnetic permeability for SFM materials $\left(\mu_{r}=350000\right)$ which has been observed in pure Iron ${ }^{66-68}$. Therefore, this cover the whole range of SFM materials available in the market, such as Ni, NiZn, MnZn, Si, C, and Co ferrites $\left(\mu_{r} \cong 5-15000\right)$, providing the first known map of AC-losses for rounded SC-SFM wires, which reveals a saturation of the hysteresis losses caused by the SFM at about $\mu_{r}=1000$, with even less than $1 \%$ difference from $\mu_{r}=100$, although showing a very rapid change in the hysteresis curve between $\mu_{r}=1$ and $\mu_{r}=100$. This is a remarkable result because it discloses that no matter the SFM used, beyond a relative magnetic permeability of $\mu_{r}=100$, nearly no increment of the AC-losses of the system could be observed by effect of the coupling between the SC and the SFM. Also, the structure of this curve reveals that the dominant factor in the hysteresis losses provided by this coupling, comes from the factor $\bar{R}_{\mu 1}$ in Eq. 13 (see Fig. 4 (c)), which also dominates the contribution to the magnetic field outside of the SC-SFM metastructure in Eq. 18 created by the locally induced profile of current $J_{i}$. This has strong implications in other phenomena such as magnetic shielding and magnetic cloaking effects by SC-SFM metastructures, where an external magnetic field $\mathbf{B}_{0}$ can be entered by the additional vector potential $\mathbf{A}_{0}\left(\mathbf{r}_{i}\right)=\mathbf{B}_{0} \times \mathbf{r}_{i}$, as still these phenomena depend on the screening properties of the SC material, i.e, on the occurrence of local profiles of current density $\mathbf{J}_{i}$, whose coupling with the SFM remains defined by the vector potentials for the couple media in Eqs. 13-15. Consequently, the shielding properties of the SFM in the rounded geometry of Fig. 1 are also limited by the factor $\bar{R}_{\mu 1}$, whose dependence on the relative radius between the SC and the SFM, as well as the magnetic permeability of the SFM, shown a nearly negligible impact for magnetic permeabilities greater than $\mu_{r}=100$, somehow contrary to the intuitive thinking that by increasing the magnetic permeability of the SFM sheath or its thickness on a SC cylindrical wire or tube, then the magnetic shielding properties of this heterostructure will increase too.

\section{Concluding Remarks}

In this article, it has been shown that the counterintuitive increment in the AC losses of monocore SC-SFM heterostructures at self-field conditions can be explained under the framework of the general critical state theory ${ }^{45}$, it extended within a multipole variational approach. In this way, we have proven that the intriguing anisotropy in the magnetic flux distribution which can be observed by Magneto Optical Imaging techniques inside the superconducting core of a SC-SFM heterostructure ${ }^{39-41}$, is caused as a straightforward consequence of the magnetostatics coupling between these two materials, and without the need of considering the occurrence of edge currents, overcritical currents, or current sharing patterns which have not been experimentally observed. Thus, as long as no current sharing between the SC and the SFM is enabled, i.e., when these materials are electrically insulated, our semi-analytical model shows how despite no alteration on the distribution of current density is to be observed in the SC-SFM heterostructure, it compared with the current distribution for a bare SC, still, under the critical state regime it is possible to observe a certain amount of magnetic field in regions where no transport current is expected to flow. Likewise, the occurrence of this phenomena leads to the significant rise and drop of the magnetic field inside the SC, it being measured near the surface of the SFM, what is in good agreement with the experimental observations.

Additionally, given the previous knowledge of the flux front profile for the current distribution inside a SC rod at self-field conditions, which not only can be calculated by fully analytical methods, but also by the numerical minimization of a variational functional either with or without the SFM sheath, we reported here fully analytical solutions for the magnetic vector potential and the magnetic field vector at any region of the space for SC-SFM cylindrical heterostructures of arbitrary dimensions. With these, a direct prove on the magneto-coupling physical mechanism that gives place to the field deformations inside SC-SFM heterostructures, these being observed by magneto optical imaging techniques $39,41,43,69$, has been provided. Likewise, the cause of the intriguing increment in the AC-losses in SC-SFM heterostructures ${ }^{36-38}$, is explained as result of the found magneto-steady coupling between the SC and the SFM sheath. Thus, we have proven that the sole coupling between the SC and a SFM sheath is capable to produce a slight rise in the AC-losses at the SC material, even when the SFM does not add any electrical nor magnetic losses to the system. Likewise, it has been found how the selection of the SFM magnetic properties, i.e., its relative magnetic permeability, can affect the most important observable macroscopic quantities such as the magnetic field created by the SC-SFM metastructure, and its energy losses. Remarkably, we have found that no matter the SFM used, nor its dimensions, for relative magnetic permeabilities $\mu_{r} \gtrsim 100$, nearly no further increment of the AC-losses could be observed by effect of the coupling between the SC and the SFM, as the curve of losses is dominated by the factor $\bar{R}_{\mu 1}$ in Eq. 13, disclosing in this way the most complete map of ac-losses up to date for SC-SFM rounded heterostructures. Thence, the electromagnetic 
phenomena shown in this paper can be used as a benchmark to understand other technologies which can use SC-SFM rounded metastructures such as in lossless three phase power cables and high energies accelerator magnets.

\section{References}

1. Glowacki, B. \& Majoros, M. Superconducting-magnetic heterostructures: a method of decreasing ac losses and improving critical current density in multifilamentary conductors. J. Physics: Condens. Matter 21, 254206 (2009).

2. Sanchez, A., Navau, C., Prat-Camps, J. \& Chen, D.-X. Antimagnets: controlling magnetic fields with superconductormetamaterial hybrids. New J. Phys. 13, 093034 (2011).

3. Navau, C., Prat-Camps, J., Romero-Isart, O., Cirac, J. I. \& Sanchez, A. Long-distance transfer and routing of static magnetic fields. Phys. review letters 112, 253901 (2014).

4. Solovyov, M., Šouc, J. \& Gömöry, F. Magnetic cloak for low frequency ac magnetic field. IEEE Transactions on Appl. Supercond. 25, 1-5, DOI: 10.1109/TASC.2014.2376176 (2015).

5. Genenko, Y. A., Rauh, H. \& Kurdi, S. Finite-element simulations of hysteretic alternating current losses in a magnetically coated superconducting tubular wire subject to an oscillating transverse magnetic field. J. Appl. Phys. 117, 243909, DOI: 10.1063/1.4922982 (2015).

6. Gömöry, F. et al. Experimental realization of a magnetic cloak. Science 335, 1466-1468 (2012).

7. Gömöry, F., Solovyov, M. \& Šouc, J. Magnetization loop modelling for superconducting/ferromagnetic tube of an ac magnetic cloak. Supercond. Sci. Technol. 28, 044001 (2015).

8. Šouc, J., Solovyov, M. \& Gömöry, F. Hiding objects in ac magnetic fields of power grid frequency by two-shell ferromagnetic/superconducting cloak. Appl. Phys. Lett. 109, 033507, DOI: 10.1063/1.4959581 (2016).

9. Peña-Roche, J., Genenko, Y. A. \& Badía-Majós, A. Magnetic invisibility of the magnetically coated type-ii superconductor in partially penetrated state. Appl. Phys. Lett. 109, 092601, DOI: 10.1063/1.4961672 (2016).

10. Fareed, M. U., Robert, B. C. \& Ruiz, H. S. Electric field and energy losses of rounded superconducting/ferromagnetic heterostructures at self-field conditions. IEEE Transactions on Appl. Supercond. 29, 5900705, DOI: 10.1109/TASC.2019. 2893896 (2019).

11. Baghdadi, M., Ruiz, H. S. \& Coombs, T. A. Nature of the low magnetization decay on stacks of second generation superconducting tapes under crossed and rotating magnetic field experiments. Sci. Reports 8, 1342, DOI: 10.1038/ s41598-018-19681-8 (2018).

12. Genenko, Y. A., Yampolskii, S. V. \& Pan, A. V. Virgin magnetization of a magnetically shielded superconductor wire: Theory and experiment. Appl. Phys. Lett. 84, 3921-3923, DOI: 10.1063/1.1741036 (2004).

13. Pang, C., Campbell, A. \& McLaren, P. Losses in nb/ti multifilamentary composite when exposed to transverse alternating and rotating fields. IEEE Transactions on Magn. 17, 134-137, DOI: 10.1109/TMAG.1981.1061025 (1981).

14. Kirchmayr, H. R. Permanent magnets and hard magnetic materials. J. Phys. D: Appl. Phys. 29, 2763 (1996).

15. Itoh, M., Mori, K. \& Minemoto, T. Magnetic shielding effects by the superposition of a six-layered ferromagnetic cylinder over a bpscco cylinder. IEEE Transactions on Magn. 32, 2605-2608 (1996).

16. Lousberg, G. P., Fagnard, J., Ausloos, M., Vanderbemden, P. \& Vanderheyden, B. Numerical study of the shielding properties of macroscopic hybrid ferromagnetic/superconductor hollow cylinders. IEEE Transactions on Appl. Supercond. 20, 33-41, DOI: 10.1109/TASC.2009.2036855 (2010).

17. Lousberg, G. P. et al. Magnetic properties of drilled bulk high-temperature superconductors filled with a ferromagnetic powder. Supercond. Sci. Technol. 24, 035008 (2011).

18. Horvat, J., Yeoh, W. K., Kim, J. H. \& Dou, S. X. Transport and magnetic critical current in superconducting mgb2 wires. Supercond. Sci. Technol. 21, 065003 (2008).

19. Horvat, J., Soltanian, S. \& Yeoh, W. K. The relevance of the self-field for the 'peak effect' in the transport j c ( h ) of iron-sheathed mgb 2 wires. Supercond. Sci. Technol. 18, 682 (2005).

20. Kováč, P., Hušek, I., Melišek, T., Kulich, M. \& Štrbík, V. Mgb2 composite wires with fe, nb and ta sheaths. Supercond. Sci. Technol. 19, 600 (2006).

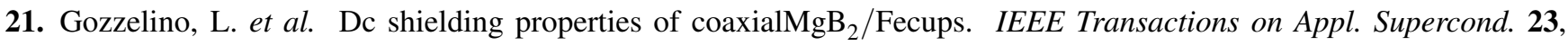
8201305-8201305, DOI: 10.1109/TASC.2012.2234817 (2013). 
22. Gozzelino, L., Gerbaldo, R., Ghigo, G., Laviano, F. \& Truccato, M. Comparison of the shielding properties of superconducting and superconducting/ferromagnetic bi- and multi-layer systems. J. Supercond. Nov. Magn. 30, 749-756, DOI: 10.1007/s10948-016-3659-z (2017).

23. Genenko, Y. A., Snezhko, A. \& Freyhardt, H. C. Overcritical states of a superconductor strip in a magnetic environment. Phys. Rev. B 62, 3453-3472, DOI: 10.1103/PhysRevB.62.3453 (2000).

24. Genenko, Y., Usoskin, A., Snezhko, A. \& Freyhardt, H. Overcritical states in magnetically shielded superconductor strips. Phys. C: Supercond. 341-348, 1063 - 1064, DOI: 10.1016/S0921-4534(00)00784-X (2000).

25. Genenko, Y. A. \& Snezhko, A. V. Superconductor strip near a magnetic wall of finite thickness. J. Appl. Phys. 92, 357-360, DOI: $10.1063 / 1.1480112(2002)$.

26. Genenko, Y. A. Overcritical states of a superconductor strip in all-superconducting environments. Phys. Rev. B 66, 184520, DOI: 10.1103/PhysRevB.66.184520 (2002).

27. Genenko, Y. A. \& Rauh, H. Enhancement of the current in a superconductor strip by means of curved superconducting shields. Appl. Phys. Lett. 82, 2115-2117, DOI: 10.1063/1.1560866 (2003).

28. Genenko, Y. A. \& Rauh, H. Novel superconductor/magnet resonant configurations: Exact analytic representations of the meissner state and the critical state. J. Physics: Conf. Ser. 43, 568 (2006).

29. Yampolskii, S., Genenko, Y. \& Rauh, H. Penetration of an external magnetic field into a multistrip superconductor/softmagnet heterostructure. Phys. C: Supercond. 460-462, 1262 - 1263, DOI: 10.1016/j.physc.2007.04.065 (2007). Proceedings of the 8th International Conference on Materials and Mechanisms of Superconductivity and High Temperature Superconductors.

30. Genenko, Y. A., Rauh, H. \& Krüger, P. Finite-element simulations of hysteretic ac losses in a bilayer superconductor/ferromagnet heterostructure subject to an oscillating transverse magnetic field. Appl. Phys. Lett. 98, 152508, DOI: 10.1063/1.3560461 (2011).

31. Genenko, Y. A. Magnetic shielding for improvement of superconductor performance. Phys. Status Solidi (a) 189, 469 (2002).

32. Yampolskii, S., Genenko, Y. \& Rauh, H. Distribution of the sheet current in a magnetically shielded superconducting filament. Phys. C: Supercond. 415, 151 - 157, DOI: 10.1016/j.physc.2004.05.012 (2004).

33. Genenko, Y. A., Rauh, H. \& Yampolskii, S. V. The bean-livingston barrier at a superconductor/magnet interface. J. Physics: Condens. Matter 17, L93 (2005).

34. Yampolskii, S. V. \& Genenko, Y. A. Entry of magnetic flux into a magnetically shielded type-ii superconductor filament. Phys. Rev. B 71, 134519, DOI: 10.1103/PhysRevB.71.134519 (2005).

35. Yampolskii, S. V., Genenko, Y. A., Rauh, H. \& Snezhko, A. V. The bean model of the critical state in a magnetically shielded superconductor filament. J. Physics: Conf. Ser. 43, 576 (2006).

36. Eckelmann, H., Däumling, M., Quilitz, M. \& Goldacker, W. Ac transport current losses of multifilamentary bi(2223) tapes with varying filament geometries. Phys. C, Supercond. 295, 198-208 (1998).

37. Huang, Y. B. et al. Development of bi(2223) multifilamentary tapes with low ac losses. J. Supercond. Incorporating Nov. Magn. 11, 495-505 (1998).

38. Majoros, M., Glowacki, B. \& Campbell, A. Magnetic screening as a possible way to decrease transport ac losses in multifilamentary superconductors - basic theoretical considerations. Phys. C: Supercond. 334, 129 - 140, DOI: https://doi.org/10.1016/S0921-4534(00)00276-8 (2000).

39. Pan, A. V., Zhou, S., Liu, H. \& Dou, S. Direct visualization of iron sheath shielding effects in MgB2superconducting wires. Supercond. Sci. Technol. 16, L33-L36, DOI: 10.1088/0953-2048/16/10/101 (2003).

40. Pan, A. V., Dou, S. \& H., J. T. Magneto-optical imaging of magnetic screening in superconducting wires. In MagnetoOptical Imaging. NATO Science Series (Series II: Mathematics, Physics and Chemistry), vol. 142, DOI: https://doi.org/10. 1007/978-94-007-1007-8_18 (Springer, Dordrecht, 2004).

41. Roussel, M., Pan, A. V., Zhou, S. \& Dou, S. X. Influence of the iron sheath on the local supercurrent distribution in MgB2wires. J. Physics: Conf. Ser. 43, 95-98, DOI: 10.1088/1742-6596/43/1/024 (2006).

42. Horvat, J., Wang, X. L., Soltanian, S. \& Dou, S. X. Improvement of critical current in mgb2/fe superconducting wires by a ferromagnetic sheath. Appl. Phys. Lett. 80, 829-831, DOI: 10.1063/1.1447010 (2002). 
43. Roussek, M. Magneto-optical imaging in superconductors, PhD Thesis (Faculty of Engineering, University of Wollongong Theses, Wollongong, Australia, 2007).

44. Wells, F. S. Magneto-optical imaging and current profiling on superconductors, PhD Thesis (Faculty of Engineering, University of Wollongong Theses, Wollongong, Australia, 2011).

45. Badía-Majós, A., López, C. \& Ruiz, H. S. General critical states in type-II superconductors. Phys. Rev. B 80, 144509, DOI: 10.1103/PhysRevB.80.144509 (2009).

46. Robert, B. C., Fareed, M. U. \& Ruiz, H. S. How to choose the superconducting material law for the modelling of $2 \mathrm{~g}$-hts coils. Materials 12, 2679, DOI: 10.3390/ma12172679 (2019).

47. Gurevich, A. V., Mints, R. G. \& Rakhmanov, A. L. Physics of Composite Superconductors (Begell House, New York, 1997).

48. Grilli, F. Numerical modeling of hts applications. IEEE Transactions on Appl. Supercond. 26, 0500408, DOI: 10.1109/ TASC.2016.2520083 (2016).

49. Devred, A. Review of superconducting dipole and quadrupole magnets for particle accelerators. In CERN Accelerator School (CAS 97): Measurement and Alignment of Accelerator and Detector Magnets, 43-78 (1998).

50. Devred, A. Superconducting magnets for particle accelerators and storage rings (Wiley Encyclopedia of Electrical and Electronic Engineering, 1999).

51. Devred, A. 1999 review of superconducting dipole and quadrupole magnets for particle accelerators. In CEA Saclay, Dept. d'Astrophysique de la Physique des Particules de la Physique Nucleaire et de l'Instrumentation Associee (DAPNIA), 162 (1999).

52. Devred, A. \& Trassart, D. Magnetic field produced by a single current line near or within a ferromagnetic cylinder or tube. In European Organization for Nuclear Research, Laboratory for Particle Physics (2005).

53. Badía, A. \& López, C. Critical state theory for nonparallel flux line lattices in type-II superconductors. Phys. Rev. Lett. 87, 127004, DOI: 10.1103/PhysRevLett.87.127004 (2001).

54. Badía, A. \& López, C. Vector magnetic hysteresis of hard superconductors. Phys. Rev. B 65, 104514, DOI: 10.1103/ PhysRevB.65.104514 (2002).

55. Ruiz, H. S., Badía-Majós, A. \& López, C. Material laws and related uncommon phenomena in the electromagnetic response of type-II superconductors in longitudinal geometry. Supercond. Sci. Technol. 24, 115005 (2011).

56. Ruiz, H. S., Badía-Majós, A., Genenko, Y. A., Rauh, H. \& Yampolskii, S. V. Superconducting wire subject to synchronous oscillating excitations: Power dissipation, magnetic response, and low-pass filtering. Appl. Phys. Lett. 100, 112602, DOI: 10.1063/1.3693614 (2012).

57. Ruiz, H. S., Badía-Majós, A., Genenko, Y. A. \& Yampolskii, S. V. Strong localization of the density of power losses in type-II superconducting wires. IEEE Transactions on Appl. Supercond. 23, 8000404-8000404, DOI: 10.1109/TASC.2012. 2232695 (2013).

58. Ruiz, H. S. \& Badía-Majós, A. Exotic magnetic response of superconducting wires subject to synchronous and asynchronous oscillating excitations. J. Appl. Phys. 113, 193906, DOI: 10.1063/1.4804931 (2013).

59. Robert, B. C. \& Ruiz, H. S. Electromagnetic response of dc type-ii superconducting wires under oscillating magnetic excitations. IEEE Transactions on Appl. Supercond. 28, 8200905, DOI: 10.1109/TASC.2017.2668060 (2018).

60. Robert, B. C. \& Ruiz, H. S. Magnetization profiles of ac type-ii superconducting wires exposed to dc magnetic fields. IEEE Transactions on Appl. Supercond. 28, 8200805, DOI: 10.1109/TASC.2018.2794138 (2018).

61. Robert, B. C. \& Ruiz, H. S. Magnetic characteristics and ac losses of dc type-ii superconductors under oscillating magnetic fields. Supercond. Sci. Technol. (2018).

62. Vlasko-Vlasov, V. K. et al. Self-healing patterns in ferromagnetic-superconducting hybrids. Supercond. Sci. Technol. 28, 035006, DOI: 10.1088/0953-2048/28/3/035006 (2015).

63. Bean, C. P. Magnetization of hard superconductors. Phys. Rev. Lett. 8, 250-253, DOI: 10.1103/PhysRevLett.8.250 (1962).

64. Ruiz, H. S., López, C. \& Badía-Majós, A. Inversion mechanism for the transport current in type-II superconductors. Phys. Rev. B 83, 014506, DOI: 10.1103/PhysRevB.83.014506 (2011).

65. Ruiz Rondan, H. Material laws and numerical methods in applied superconductivity (University of Zaragoza Press, Zaragoza, Spain, 2013). 
66. Williams, B. Power Electronics: Devices, Drivers, Applications, and Passive Components (McGraw-Hill, 1992).

67. DOD. Military handbook. grounding, bonding, and shielding for electronic equipments and facilities. Tech. Rep., Department of Defense, Washington, DC. (1987). MIL-HDBK-419A.

68. Solymar, L., Solymar, P., Walsh, D. \& Walsh, B. Lectures on the Electrical Properties of Materials. Oxford science publications (Oxford University Press, 1988).

69. Pan, A. V. \& Dou, S. Overcritical state in superconducting round wires sheathed by iron. J. Appl. Phys. 96, 1146-1153, DOI: $10.1063 / 1.1763224$ (2004).

\section{Acknowledgements}

H.S.R. thanks A. Badía-Majos and Y. Genenko for the valuable discussions around SC-SFM metastructures. M.U.F. thanks for the funding support received by the College of Science and Engineering of the University of Leicester. This work was supported by the UK Research and Innovation, Engineering and Physical Sciences Research Council (EPSRC), through the grant Ref. EP/S025707/1 led by H.S.R. All authors acknowledge the use of the High Performance Computing facility ALICE at the University of Leicester.

\section{Author contributions statement}

H.S.R conceived the physics and integral formulation for the numerical solution of SC/SFM metastructures into the critical state regime, and secured the funding. M.U.F. performed extensive coding implementation and software development tasks for the computation, analysis, and interpretation of data. M.U.F. and H.S.R. contributed in the analysis and writing of the manuscript. All authors have read and approved the final manuscript.

\section{Additional information}

\section{Competing financial interests}

The authors declare to have no competing interests as defined by Nature Publishing Group, or other interests that might be perceived to influence the results and/or discussion reported in this paper. 


\section{Figures}

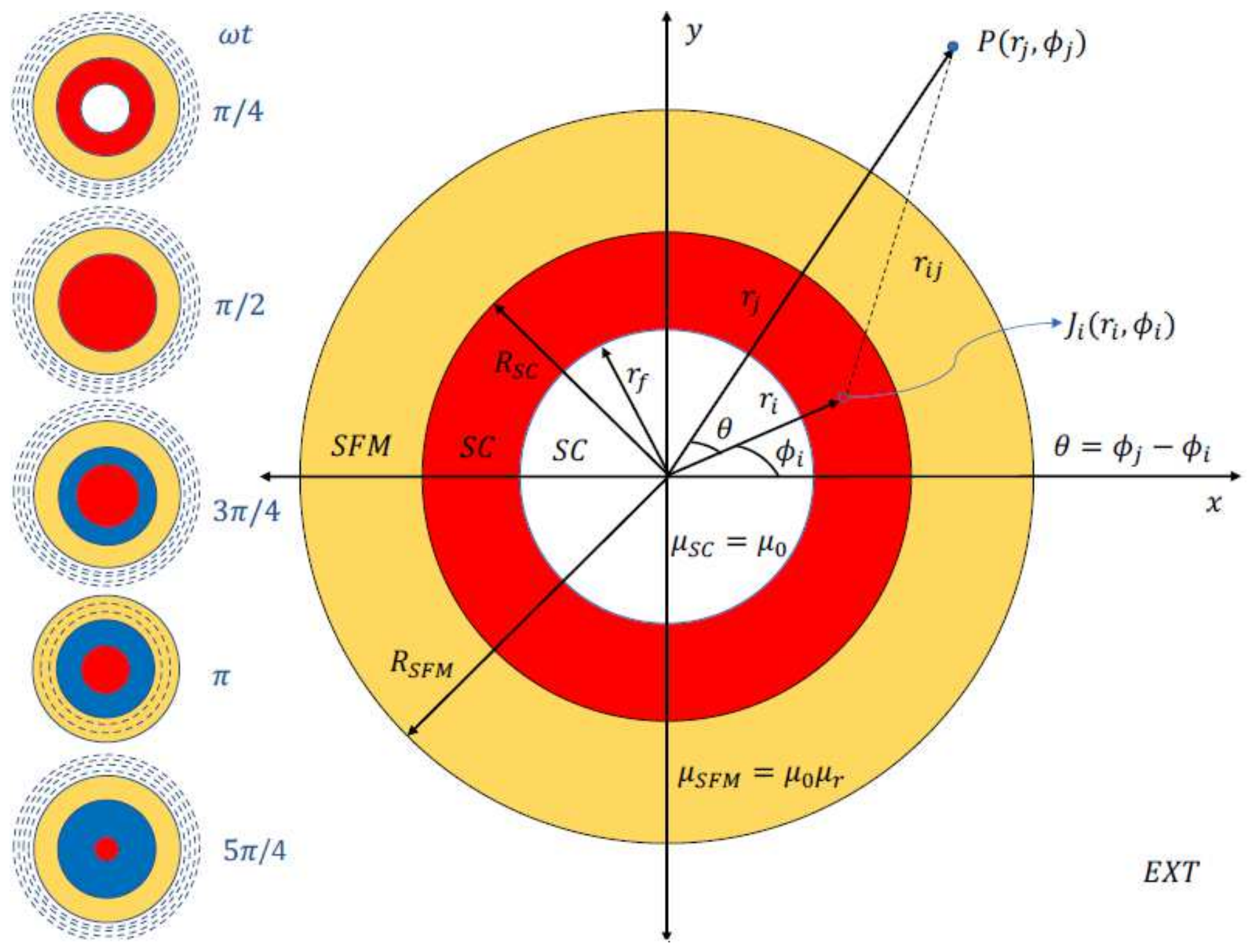

Figure 1

Pictorial representation of the cylindrical Superconducting (SC) - Soft-Ferromagnetic (SFM) metastructure analysed in this paper. of completeness. 

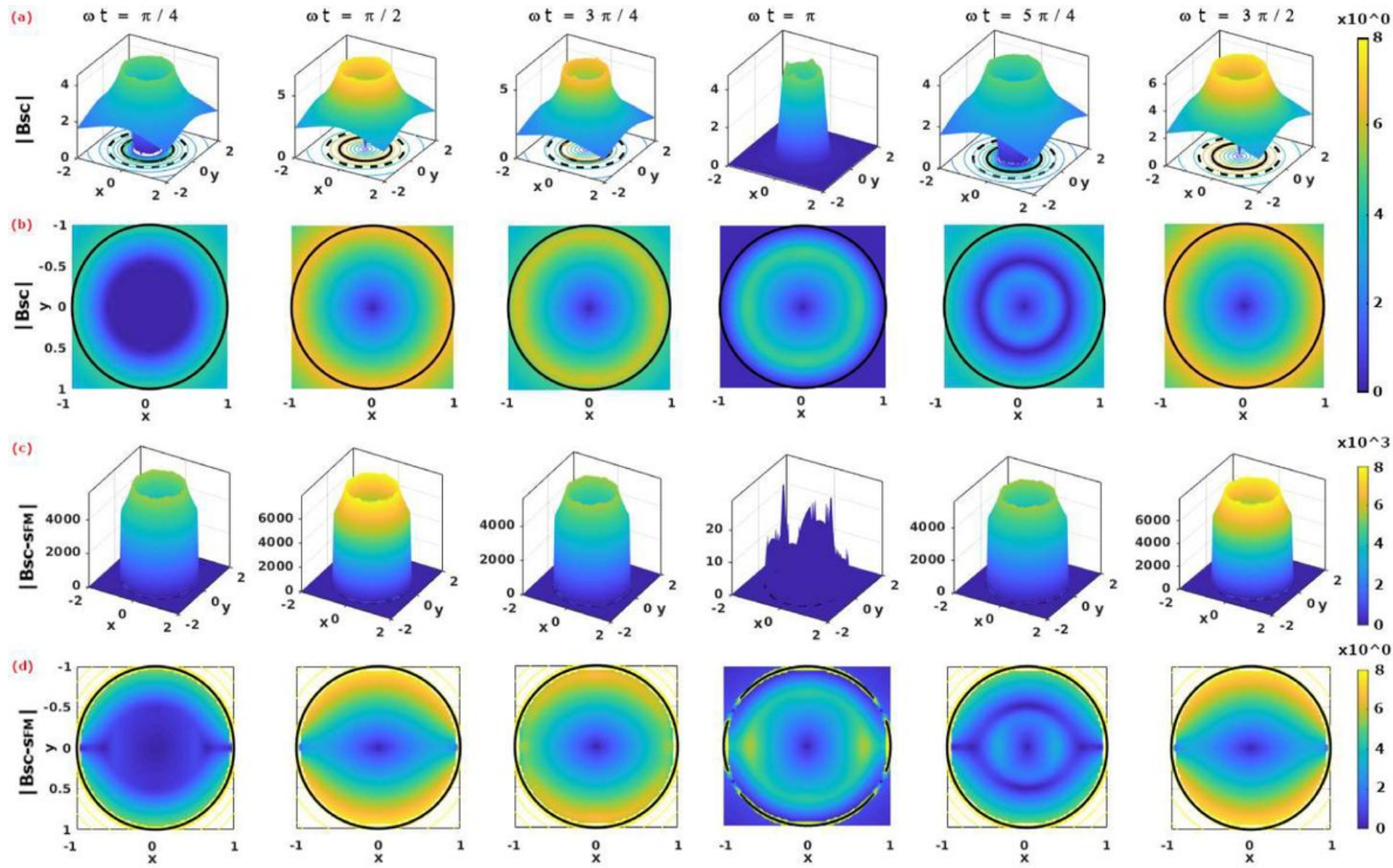

Figure 2

Dynamics of the norm of magnetic flux density 

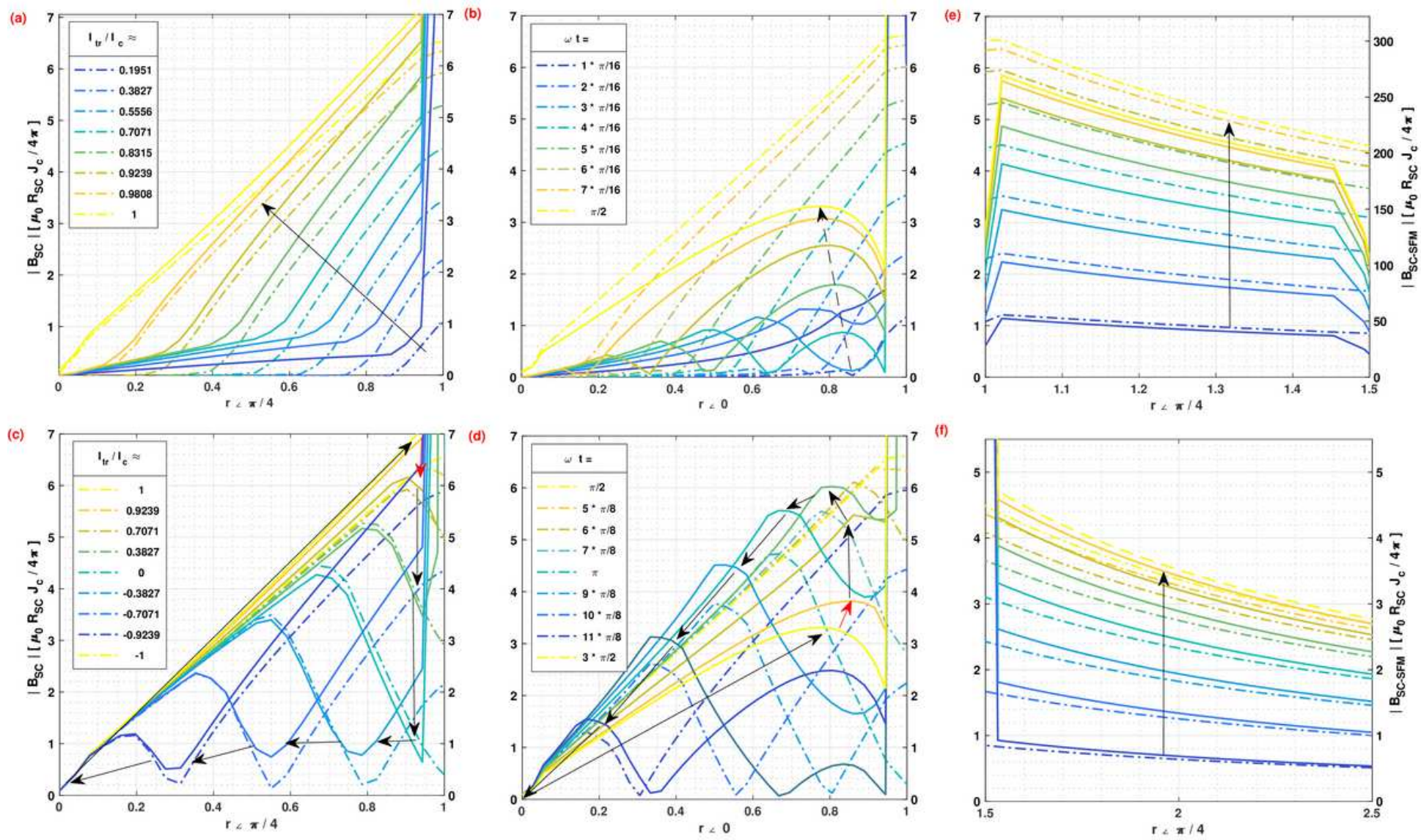

Figure 3

Dynamics of the norm of magnetic flux density over the radial directions 


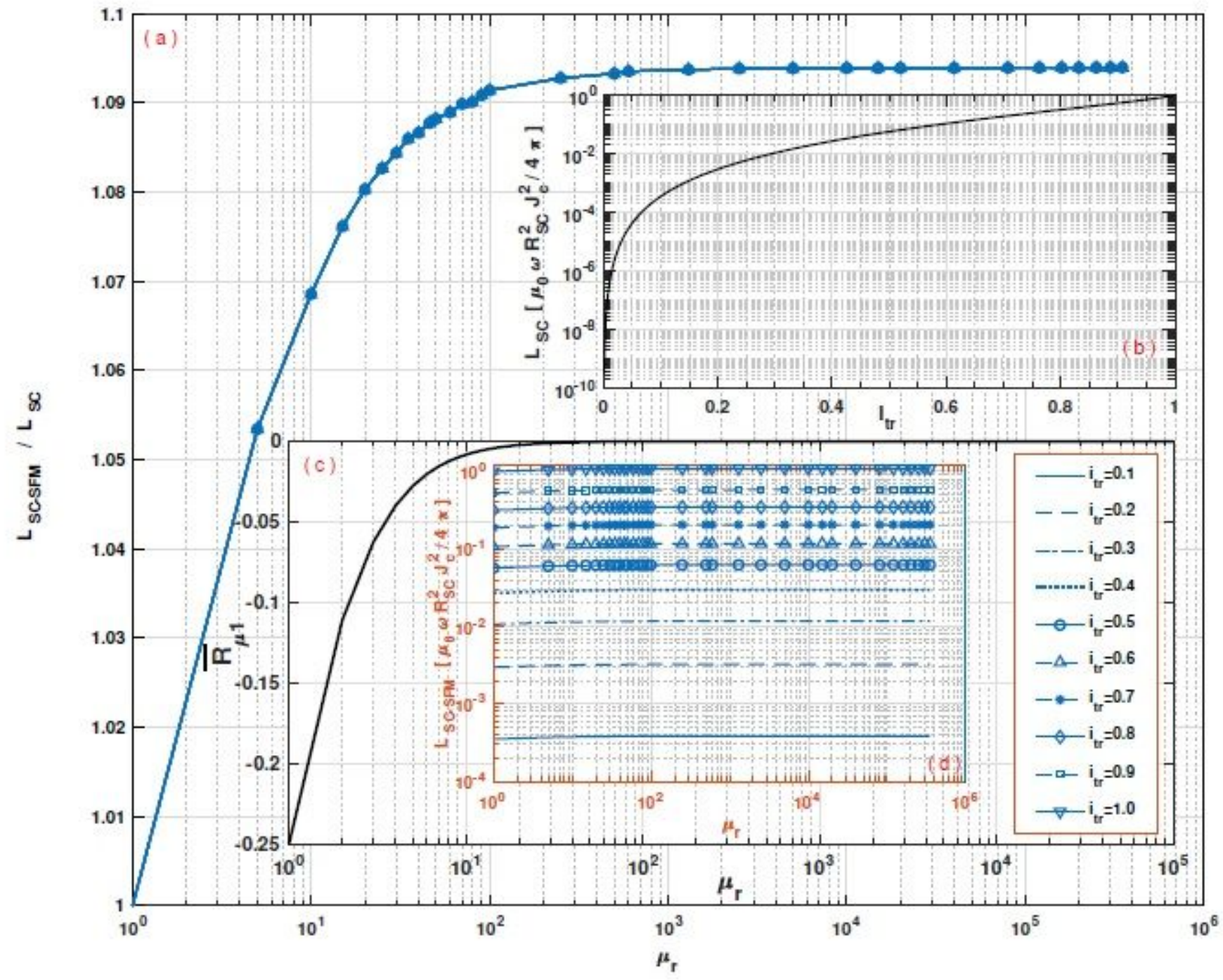

Figure 4

Hysteretic losses ratio between SC-SFM metastructures 